\title{
North American deer mice are susceptible to SARS-CoV-2
}

Bryan D. Griffin ${ }^{1}$, Mable Chan ${ }^{1}$, Nikesh Tailor ${ }^{1}$, Emelissa J. Mendoza ${ }^{1}$, Anders Leung ${ }^{1}$, Bryce M. Warner ${ }^{1,2}$, Ana T. Duggan ${ }^{3}$, Estella Moffat ${ }^{4}$, Shihua $\mathrm{He}^{1}$, Lauren Garnett ${ }^{1,2}$, Kaylie N. Tran ${ }^{1}$, Logan Banadyga ${ }^{1}$, Alixandra Albietz ${ }^{1}$, Kevin Tierney ${ }^{1}$, Jonathan Audet ${ }^{1}$, Alexander Bello ${ }^{1}$, Robert Vendramelli ${ }^{1}$, Amrit S. Boese ${ }^{1}$, Lisa Fernando ${ }^{1}$, L. Robbin Lindsay ${ }^{1,5}$, Claire M. Jardine ${ }^{6}$, Heidi Wood $^{1}$, Guillaume Poliquin ${ }^{2,7,8}$, James E. Strong ${ }^{1,2,7}$, Michael Drebot ${ }^{1,2}$, David Safronetz $^{1,2}$, Carissa Embury-Hyatt ${ }^{4}$, Darwyn Kobasa ${ }^{1,2, *}$

\section{Affiliations:}

${ }^{1}$ Zoonotic Diseases and Special Pathogens Program, National Microbiology Laboratory, Public Health Agency of Canada, 1015 Arlington Street, Winnipeg, Manitoba, Canada R3E 3R2 ${ }^{2}$ Department of Medical Microbiology and Infectious Diseases, College of Medicine, Faculty of Health Sciences, University of Manitoba, 745 Bannatyne Avenue, Winnipeg, Manitoba, Canada R3E 0J9

${ }^{3}$ Science Technology Cores and Services, National Microbiology Laboratory, Public Health Agency of Canada, 1015 Arlington Street, Winnipeg, Manitoba, Canada R3E 3R2.

${ }^{4}$ National Centre for Foreign Animal Disease, Canadian Food Inspection Agency, 1015 Arlington Street, Winnipeg, Manitoba, Canada R3E 3M4

${ }^{5}$ Department of Entomology, University of Manitoba, Winnipeg, Manitoba, Canada R3T 2N2, ${ }^{6}$ Department of Pathobiology, Canadian Wildlife Health Cooperative, Department of Pathobiology, University of Guelph, Guelph, Ontario, Canada N1G 2W1

${ }^{7}$ Pediatrics \& Child Health, College of Medicine, Faculty of Health Sciences, University of Manitoba, 745 Bannatyne Avenue, Winnipeg, Manitoba, Canada R3E 0J9

${ }^{8}$ Office of the Scientific Director, National Microbiology Laboratories, Public Health Agency of Canada, Winnipeg, MB, Canada R3E 3R2

*Corresponding author: darwyn.kobasa@canada.ca

Keywords: Severe Acute Respiratory Syndrome-coronavirus 2, SARS-CoV-2, coronavirus disease 2019, COVID-19, pandemic, angiotensin-converting enzyme 2 (Ace2), virology, infection, host specificity, species specificity, zoonoses, zooanthroponosis, reverse zoonosis, animal reservoir, re-emergence, virus shedding, virus transmission, real-time polymerase chain reaction, Cricetidae, Mesocricetus auratus, deer mice, Peromyscus maniculatus, white footed mouse, Peromyscus leucopus 


\section{Abstract}

The zoonotic spillover of the pandemic SARS-coronavirus 2 (SARS-CoV-2) from an animal reservoir, currently presumed to be the Chinese horseshoe bat, into a naïve human population has rapidly resulted in a significant global public health emergency. Worldwide circulation of SARS$\mathrm{CoV}-2$ in humans raises the theoretical risk of reverse zoonosis events with wildlife, reintroductions of SARS-CoV-2 into permissive non-domesticated animals, potentially seeding new host reservoir species and geographic regions in which bat SARS-like coronaviruses have not historically been endemic. Here we report that North American deer mice (Peromyscus maniculatus) and some closely related members of the Cricetidae family of rodents possess key amino acid residues within the angiotensin-converting enzyme 2 (ACE2) receptor known to confer SARS-CoV-2 spike protein binding. Peromyscus rodent species are widely distributed across North America and are the primary host reservoirs of several emerging pathogens that repeatedly spill over into humans including Borrelia burgdorferi, the causative agent of Lyme disease, deer tick virus, and Sin Nombre orthohantavirus, the causative agent of hantavirus pulmonary syndrome (HPS). We demonstrate that adult deer mice are susceptible to SARS-CoV2 infection following intranasal exposure to a human isolate, resulting in viral replication in the upper and lower respiratory tract with little or no signs of disease. Further, shed infectious virus is detectable in nasal washes, oropharyngeal and rectal swabs, and viral RNA is detectable in feces and occasionally urine. We further show that deer mice are capable of transmitting SARSCoV-2 to naïve deer mice through direct contact. The extent to which these observations may translate to wild deer mouse populations remains unclear, and the risk of reverse zoonosis and/or the potential for the establishment of Peromyscus rodents as a North American reservoir for SARS-CoV-2 is unknown. Nevertheless, efforts to monitor wild, peri-domestic Peromyscus rodent populations are likely warranted as the SARS-CoV-2 pandemic progresses.

\section{Main text}

Severe acute respiratory syndrome coronavirus 2 (SARS-CoV-2) is the causative agent of coronavirus disease 2019 (COVID-19), an acute respiratory illness that emerged in Wuhan, China in late $2019^{1}$, initiating a global pandemic associated with millions of infections, hundreds of thousands of deaths, and severe social and economic disruption. SARS-CoV-2, a positivesense RNA virus of the betacoronavirus genus, is closely related to the high-consequence respiratory viruses, SARS-CoV and Middle East respiratory syndrome coronavirus (MERS$\mathrm{CoV})$. While the majority of SARS-CoV-2 infections result in asymptomatic ${ }^{2,3}$ or mild respiratory disease ${ }^{4,5}$ some individuals progress to more severe disease that can result in admittance to an intensive care unit (ICU) and/or the need for oxygen therapy or invasive mechanical ventilation and can result in serious multi-organ sequelae and death. The severity of disease and likelihood of mortality often correlates with advanced age, compromised immune status, and the pre-existence of certain cardiovascular, pulmonary, and metabolic comorbidities ${ }^{4,6}$. Human-to-human transmission of SARS-CoV-2 is believed to occur primarily through short-range respiratory droplets and aerosols emitted by infected individuals during coughing, sneezing, speaking, breathing, or as a result of aerosol-generating medical procudures $^{7}$. Individuals ranging from pre-symptomatic, asymptomatic, mildly symptomatic to fulminant symptomatic are thought to be capable of transmitting SARS-CoV-2 $2^{8,9}$. 
Like the other non-seasonal human coronaviruses, SARS-CoV ${ }^{10,11}$ and MERS-CoV ${ }^{12,13}$, SARSCoV-2 is believed to be of bat origin ${ }^{11,14,15}$ although related viruses have also been identified in wild pangolins ${ }^{16}$. Examinations of amino acid sequences of the SARS-CoV-2 receptor, angiotensin-converting enzyme 2 (Ace2), from various vertebrate species predicts that additional wild animal species may be susceptible ${ }^{17,18}$. Several experimental animal models of SARS-CoV2 infection have been reported including transgenic mice that express human Ace 2 (hAce 2$)^{19,20}$ and mouse-adapted SARS-CoV-2 ${ }^{21,22}$ as well as tree shrews ${ }^{23}$, hamsters ${ }^{24,25}$, ferrets ${ }^{26,27}$, fruit bats $^{28}$, rhesus macaques ${ }^{29}$, cynomolgus macaques ${ }^{30}$, marmosets ${ }^{30}$, and African green monkeys ${ }^{31}$ that are innately susceptible to infection with wildtype SARS-CoV-2. Experimental transmission by both direct and indirect contact has been reported for hamsters ${ }^{24,25}$, ferrets ${ }^{27}$, and domestic cats $^{26,32}$. Lastly, there have been documented instances of unintentional human-to-animal transmission (zooanthroponosis) with several species of captive or domesticated animals including tigers ${ }^{33}, \operatorname{mink}^{34}$, $\operatorname{cats}^{26,35}$, and $\operatorname{dogs}^{36}$.

101 After Syrian hamsters (Mesocricetus auratus) were identified to be a SARS-CoV-2-susceptible host species ${ }^{24,25}$, we sought to determine whether or not deer mice (Peromyscus maniculatus) were also susceptible hosts since they, like hamsters, are members of the Cricetidae family of rodents (Fig. 1a). Peromyscus species rodents are susceptible to persistent infections with and are the natural host reservoirs of several important zoonotic pathogens including: Borrelia burgdorferi, the causative agent of Lyme disease ${ }^{37,38}$, deer tick virus (DTV) ${ }^{39}$, and Sin Nombre orthohantavirus (SNV) ${ }^{40,41}$. Deer mice and closely related white-footed mice (Peromyscus leucopus) are widely distributed across North America (Fig. 1b). We examined deer mouse Ace2 for congruence with human Ace2 (hAce2) at key amino acid residues known to confer efficient binding to the SARS-CoV-2 Spike (S) (Fig. 1c) and came to a conclusion consistent with recent reports that deer mice were likely to be susceptible hosts ${ }^{17,42}$. We found that deer mouse Ace2 differed from hAce 2 S-contacting residues at four locations and that the amino acid differences were unlikely to have a detrimental effect on $\mathrm{S}$ binding efficiency ${ }^{43}$. We further found that Ace 2 belonging to the white-footed mouse had in common all but three hAce 2 S-contacting residues, and white-footed mouse Ace 2 has since been shown to bind S and confer entry to SARS-CoV-2 into Hela cells ${ }^{18}$.

117 Here, eight to thirty two-week old male or female deer mice (P. maniculatus rufinus; in-house colony) were inoculated with $10^{5} \mathrm{TCID}_{50}$ of SARS-CoV-2 by an intranasal route (i.n.) and monitored daily for clinical signs and weight loss for 21 days or necropsied at 2 and 4 day postinfection (dpi). SARS-CoV-2-exposed deer mice seldom displayed clinical signs (occasionally presenting with ruffled fur), and no animals succumbed to infection (Fig. 2a) or lost weight (Fig. $2 \mathrm{~b}$ ) at any point post-infection with no differences noted related to age. At 2 dpi high levels of viral RNA (vRNA; $\sim 10^{8}-10^{10}$ genome equivalents/g) were detected in the nasal turbinates and lungs, and infectious virus (up to $3.3 \times 10^{7} \mathrm{TCID}_{50} / \mathrm{g}$ and $1.5 \times 10^{7} \mathrm{TCID}_{50} / \mathrm{g}$ ) was detected in the nasal turbinates and lungs (Fig. 2 c-d). At 4 dpi infectious virus detected in the nasal turbinates had declined by several logs while lungs titers had declined to a lesser extent (ranging from $3 \times 10^{3}$ to $1.4 \times 10^{5} \mathrm{TCID}_{50} / \mathrm{g}$ and $6.8 \times 10^{4}$ to $3.4 \times 10^{6} \mathrm{TCID}_{50} / \mathrm{g}$, respectively). Age did not make a significant contribution to the viral burden detected in the tissues (Extended Data Fig. 1). At 2 and 4 dpi high levels of vRNA $\left(\sim 10^{5}-10^{8}\right.$ genome copies/g) were detected in the small intestine and colon tissues (Fig. 2 e-f), and infectious virus was present at a low level. To monitor viral shedding, oropharyngeal and rectal swabs were collected every 2 days from 2-8 dpi (Fig. 2g-h). High levels of vRNA $\left(\sim 10^{5}-10^{7}\right.$ genome copies $\left./ \mathrm{ml}\right)$ were detected on 2 and 4 dpi in oropharyngeal swabs, and both vRNA and infectious virus peaked at 2 dpi and declined 
thereafter (Fig. $2 \mathrm{~g})$. Moderate levels of vRNA $\left(\sim 10^{3}-10^{5}\right.$ genome copies $\left./ \mathrm{ml}\right)$ were detected on 2 and 4 dpi in rectal swabs, and vRNA peaked at 2 dpi and declined thereafter (Fig. 2h). Infectious virus in rectal swabs was detected on $2,4,6$, and 8 dpi at or near the limit of detection (Fig. 2h). Viral RNA was detected in nasal washes at 6 dpi in the four deer mice for which samples were obtained $\left(\sim 10^{5}-10^{6}\right.$ genome copies/ml) (Fig. 2i). An additional experimental group of deer mice $(\mathrm{n}=5)$ were inoculated i.n. with $10^{6}$ TCID $_{50}$ of SARS-CoV-2. In this group vRNA was detected in urine samples at 6 dpi in one deer mouse $\left(\sim 10^{4}\right.$ genome copies/ml) (Fig. $\left.2 \mathrm{j}\right)$ and feces at 4 dpi in all deer mice $\left(\sim 10^{4}-10^{6}\right.$ genome copies/g) (Fig. $\left.2 \mathrm{k}\right)$. Viral RNA in blood was detected on 1 dpi, indicating transient viremia that declined in most animals by 3 dpi (Fig. 21). Studies to examine the vectorial capacity of selected tick species such as Ixodes scapularis for SARS-CoV2 may be warranted. An additional experimental group of deer mice $(n=4)$ was inoculated i.n. with $10^{5}$ TCID $_{50}$ of SARS-CoV-2, and tissues were harvested at 21 dpi (Fig. $2 \mathrm{~m}$ ). In this group vRNA was detected in the nasal turbinates $\left(\sim 10^{5}-10^{7}\right.$ genome copies/g) and lungs $\left(\sim 10^{4}-10^{6}\right.$ genome copies/g) of all deer mice and in the small intestine of two deer mice $\left(\sim 10^{4}\right.$ genome copies/g), indicating persistence of vRNA in organs that to our knowledge has not been reported in other animal models studied thus far.

Lesions in animals infected at $10^{5} \mathrm{TCID}_{50}$ were largely absent from nasal turbinates except for mild neutrophilic infiltration in the submucosa at 4 dpi (Fig. 3a, left panel). Abundant vRNA was primarily detected in epithelial cells at 2 and 4 dpi (Fig. 3a, middle panels) and anti-genomic RNA could be detected occasionally in epithelial cells at 2 dpi only (Fig. 3a, right panels). Histopathologic examination of lung tissue revealed lesions that included perivascular and peribronchiolar infiltrations of histiocytes and neutrophils with occasional multinucleated syncytial cells (Fig. 3b, left panel). Occasional discrete foci of interstitial pneumonia were observed (Fig. 3b, left panel). Abundant vRNA was primarily detected in bronchiolar epithelial cells and occasionally in the interstitium (Fig. 3b, middle panels). Anti-genomic RNA could be detected in individual scattered bronchiolar epithelial cells (Fig. 3b, right panels). Lung lesions were largely absent at $21 \mathrm{dpi}$ although some foci of inflammation with syncytia were identified, and vRNA was not detected in the lungs by ISH despite qRT-PCR positivity (Extended Data Fig. 2).

Blood biochemistry and hematological parameters were compared for uninfected $(\mathrm{n}=5)$ and SARS-CoV-2-infected deer mice $\left(\mathrm{n}=3 ; 10^{6} \mathrm{TCID}_{50}\right)$ (Fig. 4a-e). Both groups showed similar levels of white blood cells; however, infected deer mice had reduced lymphocyte counts and elevated neutrophil counts (Fig. 4a). These data appear to recapitulate the trend in hematologic values observed in COVID-19 patients, where lymphocytopenia with elevated neutrophil counts can occur ${ }^{44}$. One out of three infected deer mice had a dramatically elevated neutrophil-tolymphocyte rate (NLR), a clinical metric that has been found to be elevated in human patients during severe COVID-19 $9^{45}$, and the NLR was significantly elevated in all infected deer mice $(P$ $<0.05$, Mann-Whitney test). The lack of a difference in alanine aminotransferase (ALT) (Fig. 4c), and the reduced values for blood albumin (ALB) (Fig. 4d) and blood urea nitrogen (BUN) (Fig. 4e), when uninfected and infected deer mice were compared suggests that infection did not cause liver and kidney impairment. Additional serum biochemical values were unremarkable (Extended Data Fig. 3). Transcriptional profiling of host cytokine expression in the lungs by realtime PCR using a previously reported panel that includes TNF $\alpha$, IL-6, IL10, and IFN $\alpha^{46}$ was carried out 2 dpi (Fig. 4f) and compared to uninfected controls $(n=6)$. TNF $\alpha$ and IL-6 mRNA expression was elevated in all infected mice (mean fold changes of $2^{2.7}$ and $2^{3.5}$, respectively). IL- 
10 mRNA expression was elevated in five out of six infected animals and reduced in one (mean fold change of $\left.2^{1.5}\right)$. In contrast, IFN $\alpha$ was elevated in two and reduced in four infected deer mice (mean fold change of $\left.2^{-0.8}\right)$. Overall, the mRNA levels of inflammatory cytokines (TNF $\alpha$, IL-6, and IL10) were slightly elevated compared to what is seen in the lungs of deer mice $(\sim 2-5$ fold increase) following experimental infection with $\mathrm{SNV}^{47}$. All five deer mice infected at $10^{5}$ TCID $_{50}$ had detectable serum IgG titers against mixed spike/nucleoprotein $(\mathrm{S} / \mathrm{N})$ antigen as assessed by ELISA at 14 dpi (OD $2.7-2.8$ at 1:100) (Fig. 4g), and neutralizing antibodies by 28 dpi (plaque reduction neutralization test (PRNT 90 ) 1:40 to 1:320) (Fig. 4h).

Transmission by direct contact was examined between SARS-CoV-2-infected and naïve deer mice (experimental design schematic, Extended Data Fig. 4). Five adult male and female deer mice, infected i.n. with $10^{6} \mathrm{TCID}_{50} \mathrm{SARS} \mathrm{CoV}-2$ were each transferred to a new cage at 1 dpi and co-housed with a single naïve contact deer mouse (1:1 ratio; zero days post-direct contact $(\mathrm{dpc})$ ). Oropharyngeal and rectal swab samples taken every other day revealed that initially one contact animal (DM7, Fig. 5 a-b) rapidly became infected and shed moderate levels of vRNA as early as $2 \mathrm{dpc}$. The level of vRNA in both swab samples from DM7 continued to increase for the next 4 (oropharyngeal swabs) or 2 (rectal swabs) days then declined. The remaining contact deer mice had detectable vRNA in oropharyngeal and rectal swab samples at low levels from 2 - 4 dpc which declined until $10 \mathrm{dpc}$ when shed vRNA was again detected in both oropharyngeal and rectal swabs from all contact deer mice (including DM7). Interestingly, despite the high challenge dose administered to donor deer mice only low amounts of infectious virus were detected in swabs (Fig. 5c-d). Infectious virus was detected in oropharyngeal and rectal swabs of contact deer mouse DM7 at $4 \mathrm{dpc}$ and an oropharyngeal swab sample from contact deer mouse DM6 at $10 \mathrm{dpc}$. An additional transmission study was carried out in the same manner and nasal wash, nasal turbinates, and lung samples were collected from contact animals at 2 and $4 \mathrm{dpc}$ (Fig. 5e-f). Viral RNA was detected in nasal wash, nasal turbinates, and the lungs of contact deer mice DM19 (2 dpc) and DM30 (4 dpc), as well as in the nasal wash of deer mouse DM17 (2 dpc), DM26 (4 dpc) and DM28 (4 dpc). IgG against mixed SARS-CoV-2 spike/nucleoprotein antigens were detected in deer mouse DM7 at $21 \mathrm{dpc}$, indicating seroconversion and confirming that direct contact transmission had occurred (Fig. 5g). A similar, but more stringent transmission study was carried out using a lower dose of virus $\left(10^{5} \mathrm{TCID}_{50}\right)$, and donor and naïve deer mice were mixed at 2 dpi (Fig. 5h-i). Oropharyngeal and rectal swab samples taken every other day revealed that contact deer mice had detectable vRNA in both oropharyngeal and rectal swab samples from 2 - 4 dpc at a low levels, which declined until 6-10 dpc after which shed vRNA was again detected in oropharyngeal swabs from four out of five and rectal swabs from three out of five contacts. At 21 dpc vRNA was detected in the oropharyngeal swabs of two of five contact and was not detected in any rectal swab samples.

The described experiments demonstrate that adult deer mice are susceptible to experimental infection with a human isolate of SARS-CoV-2, resulting in asymptomatic infection or mild disease with lesions limited to mild lung pathology, despite a high viral burden and elevated levels of inflammatory cytokines in the lungs and seroconversion. The detected RNA viremia in SARS-CoV-2-infected deer mice appears to be more transient than what is observed in deer mice experimentally infected with $\mathrm{SNV}^{48}$; however, the viral shedding and the propensity for direct contact transmission appears to be greater ${ }^{41,48}$. The relatively low amount of shed infectious virus from nasal, oral, and rectal routes consistently result in direct contact transmission from infected 
to co-housed naive deer mice. SNV is also detectable in deer mouse feces and urine, and zoonotic transmission of SNV into humans is thought to result from unintentional ingestion or inhalation of contaminated deer mouse excreta ${ }^{49}$. Additional studies should be carried out to determine if vRNA detected in the feces and occasionally urine is indicative of the presence infectious virus.

231 Concerns have justifiably been raised about the potential for reverse zoonosis, human-to-animal transmission of SARS-CoV-2 into susceptible wild animal host species ${ }^{50}$. The introduction of novel pathogens into susceptible wildlife hosts can have devastating effects on wildlife populations $^{51}$. The findings reported here suggest that the impacts of SARS-CoV-2 on infected Peromyscus species rodents are likely to be minimal; however, wild deer mouse populations may be more or less susceptible to infection than the experimentally housed animals we have described. An additional concern is the potential for zoonotic maintenance of SARS-CoV-2 in an animal reservoir and/or geographic region where SARS-like coronaviruses had not previously been endemic. The findings reported here are concerning in light of the fact that Peromyscus species rodents tolerate persistent infection with and serve as the primary reservoirs for several emerging zoonotic pathogens that spillover into humans, including Borrelia burgdorferi ${ }^{37,38}$, $\mathrm{DTV}^{39}$, and $\mathrm{SNV}^{40,41}$. It should be acknowledged that although deer mice are widely distributed in North America and can live in close proximity to humans, the actual risk of human-to-deer mouse transmission remains unknown. Further, it remains unclear how well the transmission studies we describe translate to the risk of sustained transmission within wild deer mouse populations. The theoretical risk of zoonotic transmission of SARS-CoV-2 back to humans from Peromyscus species rodents would depend on additional unknowns such as whether infectious virus is present in excreta at high enough levels to initiate human infection and whether other wild or domestic animal species might serve as an intermediary host. Efforts to monitor wild, especially peri-domestic Peromyscus rodent populations are likely warranted as the SARS-CoV2512 pandemic continues to progress.

252 Lastly, we suggest that the deer mouse model of SARS-CoV-2 infection, as a largely outbred small animal model, may prove useful for studying viral pathogenesis and for evaluating the protective efficacy of experimental vaccines and therapeutics.

\section{References}

1 Zhu, N. et al. A Novel Coronavirus from Patients with Pneumonia in China, 2019. N. Engl. J. Med., doi:10.1056/NEJMoa2001017 (2020).

2 Poletti, P. et al. Probability of symptoms and critical disease after SARS- CoV-2 infection, Preprint at: https://arxiv.org/pdf/2006.08471.pdf (2020).

3 O, B. et al. Estimating the Extent of True Asymptomatic COVID-19 and Its Potential for Community Transmission: Systematic Review and Meta-Analysis Preprint at: https://www.medrxiv.org/content/10.1101/2020.05.10.20097543v2 (2020).

4 Huang, C. et al. Clinical features of patients infected with 2019 novel coronavirus in Wuhan, China. Lancet 395, 497-506, doi:10.1016/S0140-6736(20)30183-5 (2020).

5 Guan, W. J. et al. Clinical Characteristics of Coronavirus Disease 2019 in China. N. Engl. J. Med. 382, 1708-1720, doi:10.1056/NEJMoa2002032 (2020).

6 Zhou, F. et al. Clinical course and risk factors for mortality of adult inpatients with COVID-19 in Wuhan, China: a retrospective cohort study. Lancet 395, 1054-1062, doi:10.1016/S0140-6736(20)30566-3 (2020).

7 Prather, K. A., Wang, C. C. \& Schooley, R. T. Reducing transmission of SARS-CoV-2. Science 368, 14221424, doi:10.1126/science.abc6197 (2020). 
Li, R. et al. Substantial undocumented infection facilitates the rapid dissemination of novel coronavirus (SARS-CoV-2). Science 368, 489-493, doi:10.1126/science.abb3221 (2020).

Moghadas, S. M. et al. The implications of silent transmission for the control of COVID-19 outbreaks. Proc. Natl. Acad. Sci. U. S. A., doi:10.1073/pnas.2008373117 (2020).

Guan, Y. et al. Isolation and characterization of viruses related to the SARS coronavirus from animals in southern China. Science 302, 276-278, doi:10.1126/science.1087139 (2003).

$\mathrm{Hu}$, B. et al. Discovery of a rich gene pool of bat SARS-related coronaviruses provides new insights into the origin of SARS coronavirus. PLoS Pathog. 13, e1006698, doi:10.1371/journal.ppat.1006698 (2017). Memish, Z. A. et al. Middle East respiratory syndrome coronavirus in bats, Saudi Arabia. Emerg. Infect. Dis. 19, 1819-1823, doi:10.3201/eid1911.131172 (2013).

Lau, S. K. P. et al. Receptor Usage of a Novel Bat Lineage C Betacoronavirus Reveals Evolution of Middle East Respiratory Syndrome-Related Coronavirus Spike Proteins for Human Dipeptidyl Peptidase 4 Binding. J. Infect. Dis. 218, 197-207, doi:10.1093/infdis/jiy018 (2018). Zhou, P. et al. A pneumonia outbreak associated with a new coronavirus of probable bat origin. Nature, doi:10.1038/s41586-020-2012-7 (2020).

Ge, X. Y. et al. Isolation and characterization of a bat SARS-like coronavirus that uses the ACE2 receptor. Nature 503, 535-538, doi:10.1038/nature12711 (2013).

Lam, T. T. et al. Identifying SARS-CoV-2-related coronaviruses in Malayan pangolins. Nature, doi:10.1038/s41586-020-2169-0 (2020).

Damas, J. et al. Broad Host Range of SARS-CoV-2 Predicted by Comparative and Structural Analysis of ACE2 in Vertebrates, Preprint at: https://www.ncbi.nlm.nih.gov/pubmed/32511356 (2020).

Liu, Y. et al. Functional and Genetic Analysis of Viral Receptor ACE2 Orthologs Reveals Broad Potential Host Range of SARS-CoV-2, Preprint at: https://www.biorxiv.org/content/10.1101/2020.04.22.046565v2 (2020). Bao, L. et al. The pathogenicity of SARS-CoV-2 in hACE2 transgenic mice. Nature, doi:10.1038/s41586020-2312-y (2020). Hassan, A. O. et al. A SARS-CoV-2 Infection Model in Mice Demonstrates Protection by Neutralizing Antibodies. Cell, doi:10.1016/j.cell.2020.06.011 (2020).

Dinnon, K. H. et al. A mouse-adapted SARS-CoV-2 model for the evaluation of COVID-19 medical countermeasures, Preprint at: https://www.ncbi.nlm.nih.gov/pubmed/32511406 (2020).

$\mathrm{Gu}$, H. et al. Rapid adaptation of SARS-CoV-2 in BALB/c mice: Novel mouse model for vaccine efficacy, Preprint at: https://www.biorxiv.org/content/10.1101/2020.05.02.073411v1 (2020). Zhao, Y. et al. Susceptibility of tree shrew to SARS-CoV-2 infection, Preprint at: https://www.biorxiv.org/content/10.1101/2020.04.30.029736v1 (2020). Chan, J. F. et al. Simulation of the clinical and pathological manifestations of Coronavirus Disease 2019 (COVID-19) in golden Syrian hamster model: implications for disease pathogenesis and transmissibility. Clin. Infect. Dis., doi:10.1093/cid/ciaa325 (2020). Sia, S. F. et al. Pathogenesis and transmission of SARS-CoV-2 in golden hamsters. Nature, doi:10.1038/s41586-020-2342-5 (2020).

Shi, J. et al. Susceptibility of ferrets, cats, dogs, and other domesticated animals to SARS-coronavirus 2. Science 368, 1016-1020, doi:10.1126/science.abb7015 (2020). Kim, Y. I. et al. Infection and Rapid Transmission of SARS-CoV-2 in Ferrets. Cell Host Microbe 27, 704709 e 702, doi:10.1016/j.chom.2020.03.023 (2020). Schlottau, K. et al. SARS-CoV-2 in fruit bats, ferrets, pigs, and chickens: an experimental transmission study. The Lancet Microbe, doi:10.1016/S2666-5247(20)30089-6. Munster, V. J. et al. Respiratory disease in
doi:10.1038/s41586-020-2324-7 (2020). $\mathrm{Lu}$, S. et al. Comparison of SARS-CoV-2 infections among 3 species of
https://www.biorxiv.org/content/10.1101/2020.04.08.031807v1 (2020). Woolsey, C. et al. Establishment of an African green monkey model for COVID-19, Preprint at: https://www.ncbi.nlm.nih.gov/pubmed/32511377 (2020). Halfmann, P. J. et al. Transmission of SARS-CoV-2 in Domestic Cats. N. Engl. J. Med., doi:10.1056/NEJMc2013400 (2020).
United States Department of Agricult New York. (2020). 
Oreshkova, N., Molenaar, R.-J., Vreman, S., Harders, F. \& Munnink, B. B. O. SARS-CoV2 infection in farmed mink, Netherlands, April 2020, Preprint at: https://www.biorxiv.org/content/10.1101/2020.05.18.101493v1 (2020).

35 Zhang, Q. et al. SARS-CoV-2 neutralizing serum antibodies in cats: a serological investigation, Preprint at: https://www.biorxiv.org/content/10.1101/2020.04.01.021196v1 (2020).

36 Sit, T. H. C. et al. Infection of dogs with SARS-CoV-2. Nature, doi:10.1038/s41586-020-2334-5 (2020).

37 Bosler, E. M., Ormiston, B. G., Coleman, J. L., Hanrahan, J. P. \& Benach, J. L. Prevalence of the Lyme disease spirochete in populations of white-tailed deer and white-footed mice. Yale J. Biol. Med. 57, 651659 (1984).

38 Brown, R. N. \& Lane, R. S. Natural and experimental Borrelia burgdorferi infections in woodrats and deer mice from California. J. Wildl. Dis. 30, 389-398, doi:10.7589/0090-3558-30.3.389 (1994).

39 El Khoury, M. Y. et al. Potential role of deer tick virus in Powassan encephalitis cases in Lyme diseaseendemic areas of New York, U.S.A. Emerg. Infect. Dis. 19, 1926-1933, doi:10.3201/eid1912.130903 (2013).

40 Knust, B. \& Rollin, P. E. Twenty-year summary of surveillance for human hantavirus infections, United States. Emerg. Infect. Dis. 19, 1934-1937, doi:10.3201/eid1912.131217 (2013).

41 Botten, J. et al. Persistent Sin Nombre virus infection in the deer mouse (Peromyscus maniculatus) model: sites of replication and strand-specific expression. J. Virol. 77, 1540-1550, doi:10.1128/JVI.77.2.15401550.2003 (2003).

42 Luan, J., Lu, Y., Jin, X. \& Zhang, L. Spike protein recognition of mammalian ACE2 predicts the host range and an optimized ACE2 for SARS-CoV-2 infection. Biochem. Biophys. Res. Commun. 526, 165-169, doi:10.1016/j.bbrc.2020.03.047 (2020).

43 Procko, E. The sequence of human ACE2 is suboptimal for binding the S spike protein of SARS coronavirus 2, Preprint at: https://www.biorxiv.org/content/10.1101/2020.03.16.994236v3 (2020).

44 Gong, J. et al. A Tool to Early Predict Severe 2019-Novel Coronavirus Pneumonia (COVID-19) : A Multicenter Study using the Risk Nomogram in Wuhan and Guangdong, China, Preprint at: https://www.medrxiv.org/content/10.1101/2020.03.17.20037515v1.full.pdf (2020).

45 Liu, J. et al. Neutrophil-to-Lymphocyte Ratio Predicts Severe Illness Patients with 2019 Novel Coronavirus in the Early Stage, Preprint at: https://www.medrxiv.org/content/10.1101/2020.02.10.20021584v1 (2020).

46 Oko, L. et al. Profiling helper T cell subset gene expression in deer mice. BMC Immunol 7, 18, doi:10.1186/1471-2172-7-18 (2006).

47 Schountz, T. et al. Kinetics of immune responses in deer mice experimentally infected with Sin Nombre virus. J. Virol. 86, 10015-10027, doi:10.1128/JVI.06875-11 (2012).

48 Warner, B. M. et al. Development and Characterization of a Sin Nombre Virus Transmission Model in Peromyscus maniculatus. Viruses 11, doi:10.3390/v11020183 (2019).

49 Brocato, R. L. \& Hooper, J. W. Progress on the Prevention and Treatment of Hantavirus Disease. Viruses 11, doi:10.3390/v11070610 (2019).

50 Leroy, E. M., Ar Gouilh, M. \& Brugere-Picoux, J. The risk of SARS-CoV-2 transmission to pets and other wild and domestic animals strongly mandates a one-health strategy to control the COVID-19 pandemic. One Health, 100133, doi:10.1016/j.onehlt.2020.100133 (2020).

51 Blehert, D. S. et al. Bat white-nose syndrome: an emerging fungal pathogen? Science 323, 227, doi:10.1126/science.1163874 (2009).

\section{Methods References}

52 Hall, E. The mammals of North America. (John Wiley and Sons, 1981).

53 Griffin, B. D. et al. Establishment of an RNA polymerase II-driven reverse genetics system for Nipah virus strains from Malaysia and Bangladesh. Sci Rep 9, 11171, doi:10.1038/s41598-019-47549-y (2019).

54 Corman, V. M. et al. Detection of 2019 novel coronavirus (2019-nCoV) by real-time RT-PCR. Euro Surveill 25, doi:10.2807/1560-7917.ES.2020.25.3.2000045 (2020).

55 Schountz, T., Shaw, T. I., Glenn, T. C., Feldmann, H. \& Prescott, J. Expression profiling of lymph node cells from deer mice infected with Andes virus. BMC Immunol 14, 18, doi:10.1186/1471-2172-14-18 (2013).

56 Livak, K. J. \& Schmittgen, T. D. Analysis of relative gene expression data using real-time quantitative PCR and the 2(-Delta Delta C(T)) Method. Methods 25, 402-408, doi:10.1006/meth.2001.1262 (2001). 
Mendoza, E. J., Manguiat, K., Wood, H. \& Drebot, M. Two Detailed Plaque Assay Protocols for the (2020).

58 Madeira, F. et al. The EMBL-EBI search and sequence analysis tools APIs in 2019. Nucleic Acids Res. 47, W636-W641, doi:10.1093/nar/gkz268 (2019).

59 Katoh, K. \& Standley, D. M. MAFFT multiple sequence alignment software version 7: improvements in performance and usability. Mol. Biol. Evol. 30, 772-780, doi:10.1093/molbev/mst010 (2013).

60 Castresana, J. Selection of conserved blocks from multiple alignments for their use in phylogenetic analysis. Mol. Biol. Evol. 17, 540-552, doi:10.1093/oxfordjournals.molbev.a026334 (2000).

61 Stamatakis, A. RAxML version 8: a tool for phylogenetic analysis and post-analysis of large phylogenies. Bioinformatics 30, 1312-1313, doi:10.1093/bioinformatics/btu033 (2014).

62 Darriba, D. et al. ModelTest-NG: A New and Scalable Tool for the Selection of DNA and Protein Evolutionary Models. Mol. Biol. Evol. 37, 291-294, doi:10.1093/molbev/msz189 (2020).

Figure 1: Deer mouse range and predicted susceptibility to SARS-CoV-2. a, Phylogeny showing the relationships among selected members of the Cricetidae family with mice and humans. b, Geographical distribution of deer mice (based on data from Hall, 1981) ${ }^{52}$. c, Alignment of human Ace2 (hAce2) amino acid residues known to confer efficient binding of the RBD of SARS-CoV-2 spike with the corresponding Ace 2 amino acid residues from selected members of the Cricetidae family and other naturally or experimentally susceptible host species. The physiochemical properties of the amino acids residues are indicated: non-polar (yellow), polar (green), acidic (red), and basic residues (blue).

\section{Figure 2: SARS-CoV-2 infection of adult deer mice.}

$\mathbf{a}-\mathbf{m}$ Eight to thirty two-week old female or male deer mice (P. maniculatus) were inoculated with $10^{5} \mathrm{TCID}_{50}$ or $10^{6} \mathrm{TCID}_{50}$ of SARS-CoV-2 by an intranasal route (i.n.) of administration and compared to age-matched uninfected controls. Bars indicate means. Dashed lines and dotted lines indicate the limit of detection for the TCID 50 assay and qRT-PCR assay, respectively. a-b, Kaplan-Meier curve depicting survival data (a) and weight data (b) over the course of 21 days following SARS-CoV-2 exposure (challenge dose, $10^{5} \mathrm{TCID}_{50}$ ). c-f, Infectious viral load (filled in circles, left axis) and vRNA levels (empty circles, right axis) in the (c) nasal turbinates, (d) lung, (e) small intestine, and (f) colon (challenge dose, $10^{5} \mathrm{TCID}_{50}$ ). g-h, Infectious viral load (filled in circles with bars, left axis) and viral RNA levels (empty circles, right axis) in (g) oropharyngeal swabs and (h) rectal swab samples (challenge dose, $10^{5} \mathrm{TCID}_{50}$ ). i, Viral RNA levels in nasal washes at 6 dpi (infectious dose, $10^{5} \mathrm{TCID}_{50}$ ). $\mathbf{j}-\mathbf{l}$, Viral RNA levels in the (j) urine, (k) feces, and (l) blood at the indicated times post-infection (infectious dose, $10^{6} \mathrm{TCID}_{50}$ ). $\mathbf{m}$, Viral RNA levels in the nasal turbinates, lung, and small intestines at 21 days post-infection (infectious dose, $10^{5} \mathrm{TCID}_{50}$ ). Data were collected from two independent experiments. (a-b, $\mathrm{n}=$ $6 ; \mathrm{c}-\mathrm{h}, \mathrm{n}=3 / 6$ for uninfected/infected; $\mathrm{i}-\mathrm{l}, \mathrm{n}=5 ; \mathrm{m}, \mathrm{n}=4)$.

\section{Figure 3: Histopathology and virus distribution.}

Hematoxylin/eosin (H\&E) staining (left) and in situ hybridization (ISH) using antisense probes that detect the SARS-CoV-2 genome/mRNA (middle) and sense probes that detects antigenomic RNA (right) were carried out on a) nasal turbinates and $\mathbf{b}$ ) lung tissue of uninfected and SARS-CoV-2-infected deer mice $\left(10^{5} \mathrm{TCID}_{50}\right.$ i.n. route) at 2 and 4 dpi. Positive detection of viral genomic RNA/mRNA or anti-genomic RNA is indicated by magenta staining (middle/right panels and insets). Arrows indicate perivascular infiltrations of histiocytes and neutrophils 
(black), peribronchiolar infiltrations of histiocytes and neutrophils (orange), mild neutrophilic infiltration in the submucosa (blue), occasionally observed discrete foci of interstitial pneumonia (purple), occasionally observed multinucleated syncytial cells (magenta), and anti-genomic RNA occasionally in individual scattered bronchiolar epithelial cells (green and right inset). The magnification is 20x for H\&E and 20x for ISH unless otherwise indicated. Scale bars $=100 \mu \mathrm{m}$, $50 \mu \mathrm{m}$, and $20 \mu \mathrm{m}$ for 10x, 20x, and 40x, respectively.

Figure 4: Deer mouse host response to SARS-CoV-2 infection. 2-infected deer mice (infectious dose $10^{6} \mathrm{TCID}_{50}$, i.n. route) at $3 \mathrm{dpi}$, including (a) white blood cell, lymphocyte, and neutrophil counts, (b) the neutrophil-to-lymphocyte ratio, (c) alanine aminotransferase (ALT), (d) blood albumin (ALB), and (e) blood urea nitrogen (BUN). f, $\mathrm{CoV}$-2-infected deer mice and displayed relative to age-matched mock-infected animals. Gene expression was normalized using GAPDH as a control. $\mathbf{g}$, IgG antibody response against SARSCoV-2 mixed spike and nucleoprotein $(\mathrm{S} / \mathrm{N})$ antigens were assessed by ELISA using serum collected on the indicated days post-infection (infectious dose, $10^{5} \mathrm{TCID}_{50}$ ). $\mathbf{h}$, Neutralizing antibody against SARS-CoV-2 was measured by PRNT $_{90}$ using serum collected at 28 days after SARS-CoV-2 infection (infectious dose, $10^{5}$ TCID $_{50}$ ). Dotted lines indicate the limit of detection. Bars indicate mean, error bars indicate SEM (c-e) or 95\% confidence interval (f). (a-b, $\mathrm{n}=5 / 3$ for uninfected/infected; c-e, $\mathrm{n}=5 / 4$ for uninfected/infected, $\mathrm{f}, \mathrm{n}=6, \mathrm{~g}$ - $\mathrm{h}, \mathrm{n}=5$ or 6$){ }^{*}=$ $P<0.05$, ns $=P>0.05$, Mann-Whitney test (b), unpaired t test (c-e).

Figure 5: Transmission of SARS-CoV-2 between deer mice through direct contact. a-g, Adult male and female deer mice were exposed to $10^{6}$ TCID 50 SARS-CoV-2 by an i.n. route of infection. At 1 dpi individual inoculated donor deer mice were transferred to a new cage and co-housed with a single naïve deer mouse (1:1 ratio) to assess SARS-CoV-2 transmission by direct contact. Deer mice were either maintained in direct contact throughout the 10 day study for serial swabbing or humanely euthanized on 2 or 4 days post-contact (dpc) for tissue collection. a-d, Viral RNA levels $(\mathbf{a}, \mathbf{b})$ and infectious viral loads $(\mathbf{c}, \mathbf{d})$ were measured in oropharyngeal swabs and rectal swab samples every other day from - 1 to 10 days after direct contact was initiated. Oropharyngeal or rectal swab samples obtained from the same animal are demarcated with a unique colour, and samples derived from co-housed pairs share the same symbol. e-f, An additional transmission study was carried out in the same manner and nasal wash, nasal turbinates, and lung samples were collected from contact animals at (e) 2 dpc and (f) 4 dpc. g, IgG antibody response (reciprocal serum dilution) against SARS-CoV-2 S and N in donor and direct-contact exposed animals were assessed by ELISA using serum collected at 21 dpc. h-i, In an additional study adult male and female deer mice were exposed to $10^{5} \mathrm{TCID}_{50}$ SARS-CoV-2 by an intranasal route of infection. At 2 dpi individual inoculated donor deer mice were transferred to a new cage and co-housed with a single naïve deer mouse (1:1 ratio) to assess SARS-CoV-2 transmission by direct contact $(n=5)$. Viral RNA levels were measured in (h) oropharyngeal swabs and (i) rectal swab samples every other day from 0 to 14 days after direct contact was initiated, and final swab samples were collected at $21 \mathrm{dpi}$ and assessed. Dotted or dashed lines indicate the limit of detection. Bars indicate means. Data were collected from two independent experiments. 


\section{Methods}

\section{Ethics statement}

The experiments described in this study were carried out at the National Microbiology Laboratory (NML) at the Public Health Agency of Canada as described in the Animal use document AUD\# H-20-006. Experiment were approved by the Animal Care Committee located at the Canadian Science Center for Human and Animal Health in accordance with the guidelines provided by the Canadian Council on Animal Care. All procedures were performed under anesthesia, and all efforts were made to minimize animal suffering and to reduce the number of animals used. All infectious work was performed under biosafety level 3 (BSL-3) conditions or higher.

\section{Viruses}

The SARS-CoV-2 strain used in these studies (SARS-CoV-2; hCoV- 19/Canada/ON-VIDO01/2020, GISAID accession\# EPI_ISL_425177) was isolated from a clinical specimen obtained at the Sunnybrook Research Institute (SRI)/ University of Toronto on VeroE6 cells and provided by the Vaccine and Infectious Disease Organization (VIDO) with permission. The P1 virus was subsequently passaged at a 1:1000 dilution on mycoplasma-free VeroE6 cells (ATCC) in Dulbecco's Modified Eagle's Medium (Hyclone) containing 1\% L-glutamine and $0.5 \mu \mathrm{g} / \mathrm{ml}$ of TPCK-trypsin and harvested when $80 \%$ cytopathic effect (CPE) became evident. The P2 virus stock was clarified by centrifugation at $6000 \mathrm{x}$ g for 5 minutes and stored at $-80^{\circ} \mathrm{C}$ until thawed for deer mouse infections. The virus stock was titrated on Vero cells by conventional TCID 50 assay, as described previously ${ }^{53}$.

\section{Deer mouse challenge experiments}

Deer mice (Peromyscus maniculatus rufinus) used in these studies were supplied by a breeding colony housed at the University of Manitoba in a pathogen-free facility. All the deer mice were acclimated for a minimum of one week prior to the initiation of experimental procedures. Deer mice were randomly assigned to their respective groups and were housed in a temperaturecontrolled, light-cycled facility. Deer mice were supplied with food and water ad libitum and were monitored daily throughout the course of the experiments. Thirteen to thirty two-week old male or female deer mice were infected with $10^{5}$ or $10^{6}$ TCID $_{50}$ of SARS-CoV-2 by an intranasal route (i.n.) of administration in a $50 \mu \mathrm{l}$ volume. Blinding of the animal experiments was not performed. Sample sizes were calculated a priori, and no animals were excluded from the data analysis although at times sample volumes were insufficient to allow downstream analyses.

\section{Blood, fluids, feces, swab, and tissue collection}

All deer mice were exsanguinated via cardiac puncture under deep isoflurane anesthesia prior to euthanization. Whole blood and serum was collected in BD microtainer tubes ( $\mathrm{K}_{2}$-EDTA or serum, respectively) (Becton, Dickinson and Company) as per the manufacturer's instructions. Serum samples were collected from the retro-orbital sinus under deep isoflurane sedation at 14, 21, and $28 \mathrm{dpi}$ for challenge studies and at $21 \mathrm{dpc}$ for transmission studies. Orophryngeal and rectal swabs were taken using fine tip Dryswab Fine Tip rayon swabs (MWE cat \#MW113). Harvested tissue samples for infectious assays were flash frozen and stored at $-80{ }^{\circ} \mathrm{C}$ until later use, and swab samples were placed in $1 \mathrm{ml}$ MEM supplemented with $1 \%$ heat-inactivated FBS, 1x L-glutamine, 
and $2 \mathrm{x}$ penicillin-streptomycin, flash frozen, and stored at $-80{ }^{\circ} \mathrm{C}$ until later use. Tissue samples harvested for vRNA detection were immersed in RNAlater (Ambion) $4{ }^{\circ} \mathrm{C}$ for 1 day, then stored at $-80^{\circ} \mathrm{C}$ until later use. For urine collection deer mice were gently raised from their cages using soft-tip mouse handling forceps and placed immediately onto a $35-\mathrm{mm}$ petri dish, a process that consistently allowed a minimum of $25 \mu \mathrm{l}$ of urine to be collected. Feces samples were collected fresh from the isoflurane chamber following sedation, homogenized in $0.5 \mathrm{ml}$ of $0.89 \% \mathrm{NaCl}$ per $80 \mathrm{mg}$ of feces using a Bead Ruptor Elite Bead Mill Homogenizer (Omni International) with a stainless steel bead at $4 \mathrm{~m} / \mathrm{s}$ for 30 seconds. The sample was then clarified for 20 minutes at 4000 $\mathrm{x} g$ and the supernatant was filtered through a $0.2 \mu \mathrm{m}$ filter. Viral RNA was then isolated using the QIAamp Viral RNA Mini kit (Qiagen), as per the manufacturer's instructions.

\section{Infectious virus in tissues and swab samples}

For infectious virus assays, thawed tissue samples were weighed and placed in $1 \mathrm{ml}$ of MEM supplemented with 1\% heat-inactivated FBS, 1x L-glutamine before being homogenized in a Bead Ruptor Elite Bead Mill Homogenizer (Omni International) at $4 \mathrm{~m} / \mathrm{s}$ for 30 seconds and clarified by centrifugation at $1500 \mathrm{x}$ g for 6 minutes. Swab samples were vortexed for 10 seconds and clarified at $2400 \mathrm{rpm}$ for 5 minutes. Tissue homogenates or swab samples were serially diluted 10fold in MEM supplemented with 1\% heat-inactivated FBS, 1x L-glutamine, and 2x penicillinstreptomycin. One hundred microliter volumes of neat samples (swabs only) and sample dilutions were added to 96 -well plates of $95 \%$ confluent Vero cells containing $50 \mu 1$ of the same medium in replicates of three and incubated for 5 days at $37^{\circ} \mathrm{C}$ with $5 \% \mathrm{CO}_{2}$. Plates were scored for the presence of cytopathic effect on day 5 after infection.

\section{Viral RNA copies in tissues and swab samples}

For vRNA copy number analysis, tissue samples were thawed and weighed and homogenized in $600 \mu 1$ RLT buffer using a Bead Ruptor Elite Bead Mill Homogenizer (Omni International) with a stainless steel bead at $4 \mathrm{~m} / \mathrm{s}$ for 30 seconds. Viral RNA from $30 \mathrm{mg}$ tissue samples was extracted with the RNeasy Plus Mini kit (Qiagen), and vRNA from swab samples was extracted with the QIAamp Viral RNA Mini kit (Qiagen). A SARS-CoV-2 E-specific real-time RT-PCR assay was used for the detection of vRNA ${ }^{54}$. RNA was reverse transcribed and amplified using the primers E_Sarbeco_F1 (5'- ACAGGTACGTTAATAGTTAATAGCGT-3') and E_Sarbeco_R2 (5'ATATTGCAGCAGTACGCACACA-3') and probe E_Sarbeco_P1 (5'-FAMACACTAGCCATCCTTACTGCGCTTCG-BBQ-3') using the TaqPath 1-Step Multiplex Master Mix kit (Applied Biosystems) on a QuantStudio 5 real-time PCR system (Appiled Biosystems), as per manufacturer's instructions. A standard curve was generated in parallel for each plate using synthesized DNA and used for the quantification of viral genome copy numbers. The Design and Analysis Software version 1.5.1 (ThermoFisher Scientific) was used to calculate the cycle threshold values, and a cycle threshold value $\leq 36$ for both replicates was considered positive.

\section{Blood counts and biochemistry}

Complete blood counts were carried out using a VetScan HM5 hematology system (Abaxis Veterinary Diagnostics), as per manufacturer instructions. Analysis of serum biochemistry was performed with a VetScan VS2 analyzer (Abaxis Veterinary Diagnostics), as per manufacturer instructions. 


\section{Transcriptional profiling of host responses}

570 RNA was extracted from tissues using the RNeasy plus mini kit (Qiagen) following the manufacturer's instructions. A two-step qRT-PCR reaction in triplicate was performed on a Quantstudio 5 (Applied Biosystems). Reverse transcription was carried out with the Superscript III RT first strand synthesis kit (Invitrogen) following DNase elimination of genomic DNA. The RT reaction was performed using $100 \mathrm{ng}$ of template RNA mixed with random hexamers as primers in a $20 \mu \mathrm{L}$ reaction volume for $5 \mathrm{~min}$ at $65^{\circ} \mathrm{C}$ followed by $10 \mathrm{~min}$ at $25^{\circ} \mathrm{C}$ and $50 \mathrm{~min}$ at $50{ }^{\circ} \mathrm{C}$. The qPCR reaction was carried out using the PowerUp SYBR Green Master Mix (Applied Biosystems) with $2 \mu \mathrm{L}$ of cDNA. The cycle parameters for qPCR were $2 \mathrm{~min}$ at $50{ }^{\circ} \mathrm{C}$ and $2 \mathrm{~min}$ at $95^{\circ} \mathrm{C}$ followed by 40 cycles of $3 \mathrm{~s}$ at $95{ }^{\circ} \mathrm{C}$ and $30 \mathrm{~s}$ at $60{ }^{\circ} \mathrm{C}$. The qPCR reactions were performed in a $20 \mu \mathrm{L}$ volume with oligonucleotide pairs specific for deer mouse TNF $\alpha$, IL6, IL10, IFN $\alpha^{55}$ at a concentration of $2 \mu \mathrm{M}$. The fold change in gene expression of TNF $\alpha$, IL6, IL10, IFN $\alpha$ in SARS-CoV-2-infected versus uninfected deer mice were calculated using the $\Delta \Delta \mathrm{Ct}$ method with GAPDH as a reference gene ${ }^{56}$. GraphPad Prism's multiple $t$ test was used to perform the second subtraction so as not to lose the variation of the mock animals.

\section{SARS-CoV-2-S-specific enzyme-linked immunosorbent assay (ELISA)}

586

SARS-CoV-2 spike/nucleoprotein (S/N)-specific IgG antibody responses were assessed using an in-house assay. Briefly, a 1:100 dilution (or a serial dilution) of deer mouse serum was carried out in duplicate and added to plates pre-coated with both the spike and nucleoprotein $(\mathrm{S} / \mathrm{N})$ antigens in the same assay wells. Deer mouse IgG was detected with a KPL peroxidase-labeled polyclonal goat antibodies against Peromyscus leucopus $\operatorname{IgG}(\mathrm{H}+\mathrm{L})$ (Sera Care).

\section{Virus neutralization assay}

Deer mouse serum samples were collected and stored at $-80^{\circ} \mathrm{C}$. SARS-CoV-2 stocks were titrated and used in the PRNT ${ }^{57}$. Briefly, serum was heat-inactivated at $56^{\circ} \mathrm{C}$ for 30 minutes and diluted 2-fold from 1:40 to 1:1280 in DMEM supplemented with 2\% FBS. Diluted sera was incubated with 50 plaque forming units of SARS-CoV-2 at $37^{\circ} \mathrm{C}$ and $5 \% \mathrm{CO}_{2}$ for 1 hour. The sera-virus mixtures were added to 24-well plates containing Vero E6 cells at 100\% confluence, followed by incubation at $37^{\circ} \mathrm{C}$ and $5 \% \mathrm{CO} 2$ for 1 hour. After adsorption, $1.5 \%$ carboxymethylcellulose diluted in MEM supplemented with 4\% FBS, L-glutamine, non-essential amino acids, and sodium bicarbonate was added to each well and plates were incubated at $37^{\circ} \mathrm{C}$ and 5\% CO2 for 72 hours. The liquid overlay was removed and cells were fixed with $10 \%$ neutral-buffered formalin for 1 hour at room temperature. The monolayers were stained with $0.5 \%$ crystal violet for 10 minutes and washed with $20 \%$ ethanol. Plaques were enumerated and compared to a $90 \%$ neutralization control. The PRNT-90 endpoint titre was defined as the highest serum dilution resulting in 90\% reduction in plaques. PRNT-90 titers $\geq 1: 40$ were considered positive for neutralizing antibodies.

\section{Histopathology and vRNA in situ hybridization}

Tissues were fixed in 10\% neutral phosphate buffered formalin for two to four weeks.

Subsequently, routine processing was carried out and tissue samples were sectioned at $5 \mu \mathrm{m}$. A set of slides was stained with hematoxylin and eosin for histopathologic examination. RNA in situ hybridization (ISH) was carried out using RNAscope 2.5 HD Detection Reagent-Red (Advanced Cell Diagnostics), according to the manufacturer's instructions. Briefly, formalinfixed, paraffin-embedded tissue samples of the various tissues were mounted on slides, baked in 
a dry oven for 1 hour at $60^{\circ} \mathrm{C}$, and deparaffinized. Tissue sections were then pre-treated with RNAscope $\mathrm{H}_{2} \mathrm{O}_{2}$ to block endogenous peroxidases for 10 minutes at room temperature. Target retrieval was carried out using the RNAscope Target Retrieval Reagent for 15 minutes. RNAscope Protease Plus Reagent was then applied for 15 minutes at $40{ }^{\circ} \mathrm{C}$. The probes targeting SARS-CoV-2 RNA (V-nCoV2019-S probe, ref\#848561) or anti-genomic RNA (V-nCoV2019S-sense ref\#845701) were designed and manufactured by Advanced Cell Diagnostics. The negative probe was also obtained from Advanced Cell Diagnostics (Reference \# 310034). The stained tissues were counterstained with Gills I Hematoxylin, and the final images were captured using a light microscope equipped with a digital camera.

\section{SARS-CoV-2 transmission studies}

626 Eight to thirty two-week old female or male deer mice were challenged with $10^{5}$ or $10^{6}$ TCID $_{50}$ of SARS-CoV-2 by an intranasal route (i.n.) of administration in a $50 \mu \mathrm{l}$ volume. At 24 or 48 hpi (for $10^{6} \mathrm{TCID}_{50}$ and $10^{5} \mathrm{TCID}_{50}$, respectively) experimentally infected deer mice were placed into a fresh cage with a naïve cage mate to assess direct contact transmission (donor:contacts at 1:1 ratio). Deer mice were monitored daily for clinical signs and/or weight loss. Oropharyngeal and rectal swab samples were taken on alternate days up to 10 DPI or 14 DPI (for $10^{6}$ TCID $_{50}$ and $10^{5} \mathrm{TCID}_{50}$, respectively), and oropharyngeal and rectal swabs as well as serum samples were collected at $21 \mathrm{dpc}$.

\section{Bioinformatic analyses}

Ace2 sequences were aligned with Clustal Omega ${ }^{58}$. For the phylogenetic analysis, mitochondrial genome sequences (see Accession codes) were aligned with MAFFT v7.46759, with regions of poor alignment trimmed with Gblocks $v 0.91 \mathrm{~b}^{60}$ resulting in a final alignment of $15,393 \mathrm{bp}$ in 115 blocks of minimum 5bp lengths. A maximum likelihood phylogeny was constructed with RAxML v8.2.12 ${ }^{61}$ using the GTR $+\mathrm{I}+\mathrm{G} 4$ substitution model as selected by modeltest-ng ${ }^{62}$.

649 Ace2 sequences: Deer Mouse (Peromyscus maniculatus bairdii; XP_006973269), White-footed

\section{Data analysis}

Results were analyzed and graphed using Prism 8 software (Graphpad Software). As appropriate, statistical analyses were performed using ANOVA with multiple comparison correction, the multiple $t$ test, or the unpaired t test with Welch's correction or Mann-Whitney test.

\section{Data availability}

660 All relevant data are available from the authors upon request. 


\section{Acknowledgements}

663 We thank Samira Mubareka of Sunnybrook Research Institute and University of Toronto for providing the clinical sample and Darryl Falzarano of Vaccine and Infectious Disease Organization - International Vaccine Centre (VIDO-InterVac) for isolation of the SARS-CoV-2 strain used in these studies. The authors also thank Michelle French, Kimberly Azaransky,

667 Stephanie Kucas and Christine DeGraff of the Veterinary Technical Services at the National

668 Microbiology Laboratory (NML) for their technical assistance during the course of this work.

669

670 The work described in this manuscript was financially supported by the Public Health Agency of Canada and the Canadian Food Inspection Agency.

\section{Author Contributions}

674

The experiments were conceived of and designed by BDG, DS, and DK. The phylogenic analysis was performed by ATD. The Ace 2 alignment analysis was carried out by BDG and EM. The virus was propagated and titered by AL, and DK. Animal husbandry, infection and sample collection was carried out by BDG, MC, NT, BMW, AA, KT, and DK. Infectious virus assays were performed by BDG and MC. Sample inactivation and RNA extractions were carried out by BDG, NT, AL, BMW, LG, KNT, RV, and ABo. Real-time RT-PCR was performed by BDG and NT. Transcriptional profiling of host responses was carried out by BMW and NT. Blood counts, biochemistry, and pathology analysis was performed and interpreted by BDG, AB, GP, JS, CEH, and DK. ELISA was developed and carried out by AL and SH. PRNT assays were developed and carried out by EM, HW, and MD. Histopathology and ISH was carried out by EMo and pathology was assessed by CEH. Statistical analysis was performed by JA. Figures were prepared by BDG, EM, and LB. Interpretation of potential impacts on wild deer mice and deer mouse ecology was done by LRL and CMJ. The initial draft of the manuscript was written by BDG and DK, with all other authors contributing to editing into the manuscript into its final form. The work was managed and supervised by BDG, MC, LF, CEH, and DK.

690 The authors declare no conflicts of interest.

\section{Extended Data}

692 Extended Data Figure 1. Viral burden in the tissues by age.

693 Extended Data Figure 2. Histological analysis of lungs at 21 days following SARS-CoV-2

694 exposure.

695 Extended Data Figure 3. Serum biochemical values.

696 Extended Data Figure 4. Schematic of deer mouse SARS-CoV-2 transmission studies 1 and 2 697 design. 

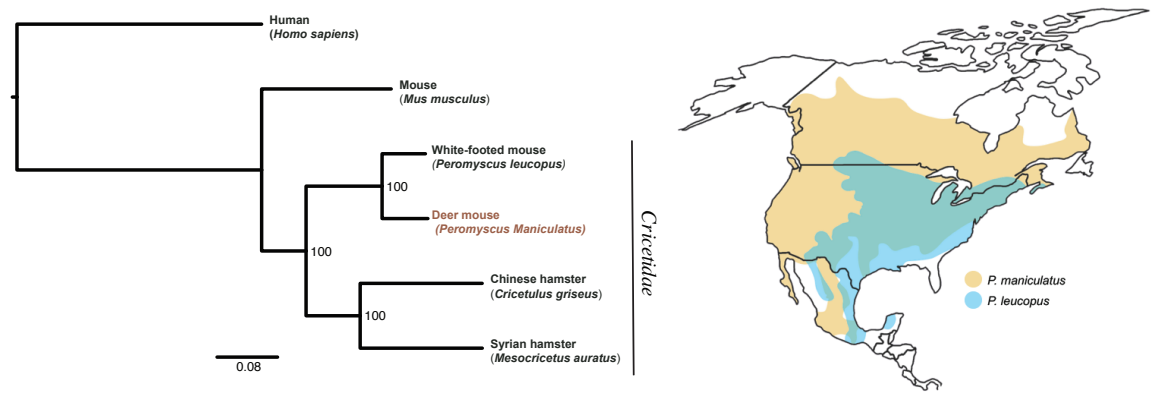

C

\begin{tabular}{|c|c|c|c|c|c|c|c|c|c|c|c|c|c|c|c|c|c|c|c|c|c|}
\hline \multirow{2}{*}{ Species } & \multirow{2}{*}{\multicolumn{19}{|c|}{ ACE2, SARS-CoV-2 spike contacting residues }} & \multicolumn{2}{|c|}{$\begin{array}{l}\text { Congruence with hAce2, } \\
\text { spike contacting residues }\end{array}$} \\
\hline & & & & & & & & & & & & & & & & & & & & AA identity & AA polarity \\
\hline Human (hAce2) & Q24 & T27 & K31 & H34 & E35 & E37 & D38 & Y41 & Q42 & L45 & L79 & M82 & Y83 & N90 & Q325 & E329 & N330 & K353 & G354 & . & . \\
\hline Syrian hamster & 0 & T & k & Q & E & E & $\theta$ & Y & 0 & L & L & N & y & N & Q & E & N & k & G & $89.5 \%(17 / 19)$ & $89.5 \%(17 / 19)$ \\
\hline Chinese hamster & a & T & k & a & E & E & D & Y & 0 & L & L & N & Y & N & Q & G & N & k & G & $84.2 \%(16 / 19)$ & $84.2 \%(16 / 19)$ \\
\hline Cat & L & $T$ & K & $\mathrm{H}$ & E & E & E & Y & Q & L & $\mathrm{L}$ & $T$ & Y & N & Q & E & N & k & G & $84.2 \%(16 / 19)$ & $89.5 \%(17 / 19)$ \\
\hline White-footed mouse & a & 1 & k & $a$ & E & E & D & Y & Q & $L$ & L & $\mathrm{N}$ & Y & N & Q & E. & N & k & G & $84.2 \%(16 / 19)$ & $84.2 \%(16 / 19)$ \\
\hline Deer mouse & 0 & 1 & K & Q & E & E & D & Y & 0 & $L$ & L & $\mathrm{N}$ & Y & $\mathrm{N}$ & Q & $\mathrm{R}$ & N & $\mathrm{k}$ & G & $78.9 \%(15 / 19)$ & $78.9 \%(15 / 19)$ \\
\hline Prairie Vole & D & A & k & Q & E & E & D & Y & 0 & $L$ & L & s & Y & N & a & E & N & k & D & $73.7 \%(14 / 19)$ & $73.7 \%(14 / 19)$ \\
\hline Fruit bat & L & $T$ & k & $T$ & E & E & D & Y & $a$ & $L$ & L & T & Y & D & E & E & k & k & G & $68.4 \%(13 / 19)$ & $68.4 \%(13 / 19)$ \\
\hline $\begin{array}{l}\text { Chinese rufous } \\
\text { horseshoe bat }\end{array}$ & $E$ & 1 & k & $T$ & k & E & $\theta$ & $\mathrm{H}$ & 0 & $L$ & L & N & Y & N & $E$ & $\mathrm{~N}$ & $\mathrm{~N}$ & k & G & $57.9 \%(11 / 19)$ & $63.2 \%(12 / 19)$ \\
\hline Masked Palm Civet & $L$ & $T$ & T & $Y$ & E & $Q$ & E & Y & Q & v & L & T & Y & $D$ & Q & E & N & k & G & $57.9 \%(11 / 19)$ & $68.4 \%(13 / 19)$ \\
\hline Ferret & L & $T$ & k & $\gamma$ & E & E & E & $Y$ & 0 & $L$ & H & $T$ & Y & D. & E & Q & $\mathrm{N}$ & k & $R$ & $52.6 \%(10 / 19)$ & $57.9 \%(11 / 19)$ \\
\hline Mouse & N & $T$ & N & a & E & E & D & $Y$ & 0 & L & T & s & $F$ & $T$ & $Q$ & A & N & $H$ & G & $526 \%(10 / 19)$ & $68.4 \%(13 / 19)$ \\
\hline
\end{tabular}

Figure 1: Deer mouse range and predicted susceptibility to SARS-CoV-2. a, Phylogeny showing the relationships among selected members of the Cricetidae family with mice and humans. b, Geographical distribution of deer mice (based on data from Hall, 1981) ${ }^{52}$. c, Alignment of human Ace2 (hAce2) amino acid residues known to confer efficient binding of the RBD of SARS-CoV-2 spike with the corresponding Ace2 amino acid residues from selected members of the Cricetidae family and other naturally or experimentally susceptible host species. The physiochemical properties of the amino acids residues are indicated: non-polar (yellow), polar (green), acidic (red), and basic residues (blue). 
Nasal Turbinates

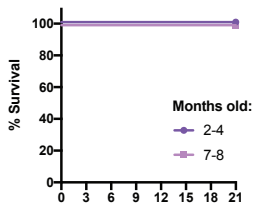

Days After Infection

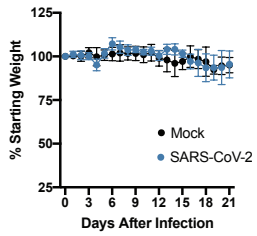

f
Colon

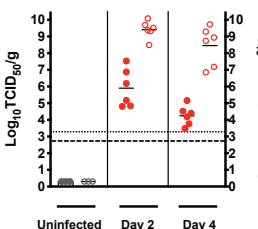

g

\section{Oropharyngeal Swabs}

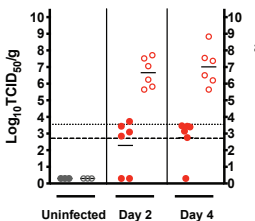

Uninfected Day 2 Day 4

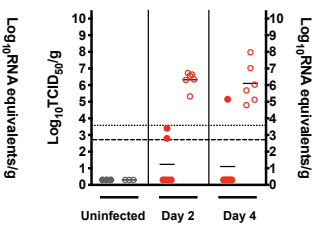

k
Nasal Wash, Day 6

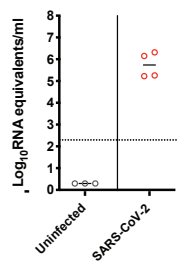

Urine, Day 6

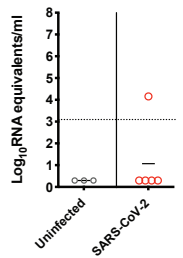

Faeces, Day 4

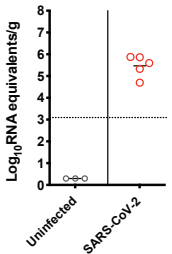

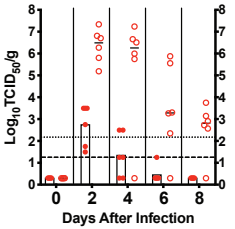

I

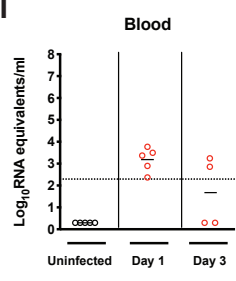

Lung

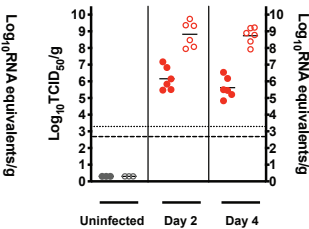

h

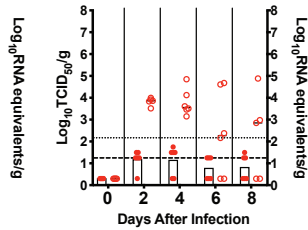

m

Day 21

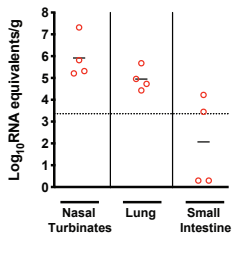

Figure 2: SARS-CoV-2 infection of adult deer mice. a-m Eight to thirty two-week old female or male deer mice (P. maniculatus) were inoculated with $10^{5} \mathrm{TCID}_{50}$ or $10^{6} \mathrm{TCID}_{50}$ of SARS-CoV-2 by an intranasal route (i.n.) of administration and compared to age-matched uninfected controls. Bars indicate means. Dashed lines and dotted lines indicate the limit of detection for the TCID $_{50}$ assay and qRT-PCR assay, respectively. a-b, Kaplan-Meier curve depicting survival data (a) and weight data (b) over the course of 21 days following SARS-CoV-2 exposure (challenge dose, $10^{5} \mathrm{TCID}_{50}$ ). c-f, Infectious viral load (filled in circles, left axis) and vRNA levels (empty circles, right axis) in the (c) nasal turbinates, (d) lung, (e) small intestine, and (f) colon (challenge dose, 10 5 $\mathrm{TCID}_{50}$ ). $\mathbf{g}$-h, Infectious viral load (filled in circles with bars, left axis) and viral RNA levels (empty circles, right axis) in (g) oropharyngeal swabs and (h) rectal swab samples (challenge dose, $10^{5}$ $\mathrm{TCID}_{50}$ ). $\mathbf{i}$, Viral RNA levels in nasal washes at 6 dpi (infectious dose, $10^{5} \mathrm{TCID}_{50}$ ). $\mathbf{j}-\mathrm{I}$, Viral RNA levels in the (j) urine, (k) feces, and (I) blood at the indicated times post-infection (infectious dose, $\left.10^{6} \mathrm{TCID}_{50}\right) . \mathrm{m}$, Viral RNA levels in the nasal turbinates, lung, and small intestines at 21 days post-infection (infectious dose, $10^{5} \mathrm{TCID}_{50}$ ). Data were collected from two independent experiments.
$(\mathrm{a}-\mathrm{b}, \mathrm{n}=6 ; \mathrm{c}-\mathrm{h}, \mathrm{n}=3 / 6$ for uninfected/infected; i-l, $\mathrm{n}=5 ; \mathrm{m}, \mathrm{n}=4)$. 

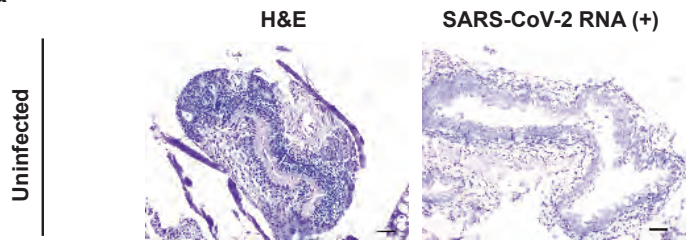

SARS-CoV-2 RNA (-)
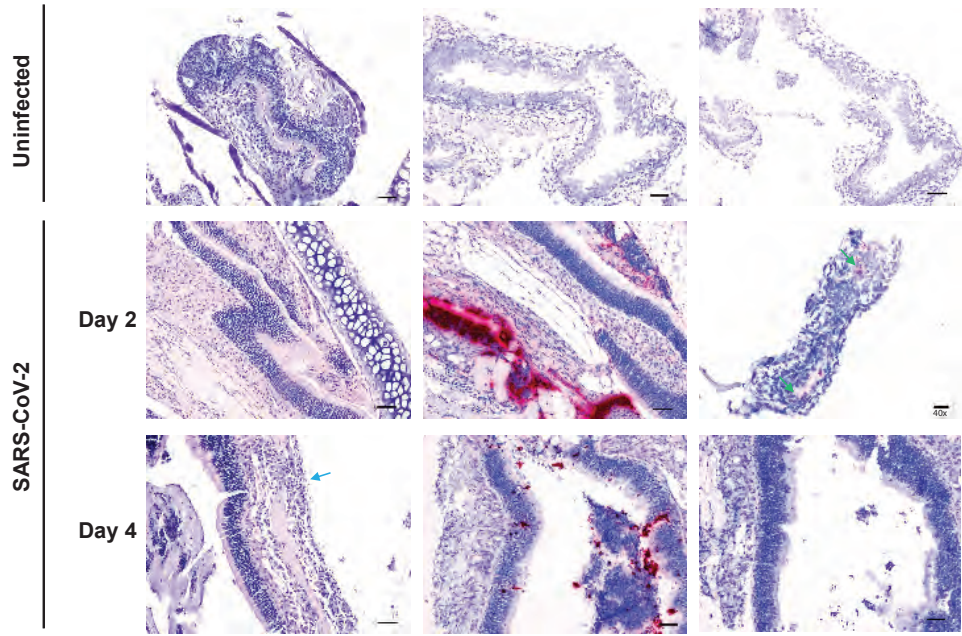

b
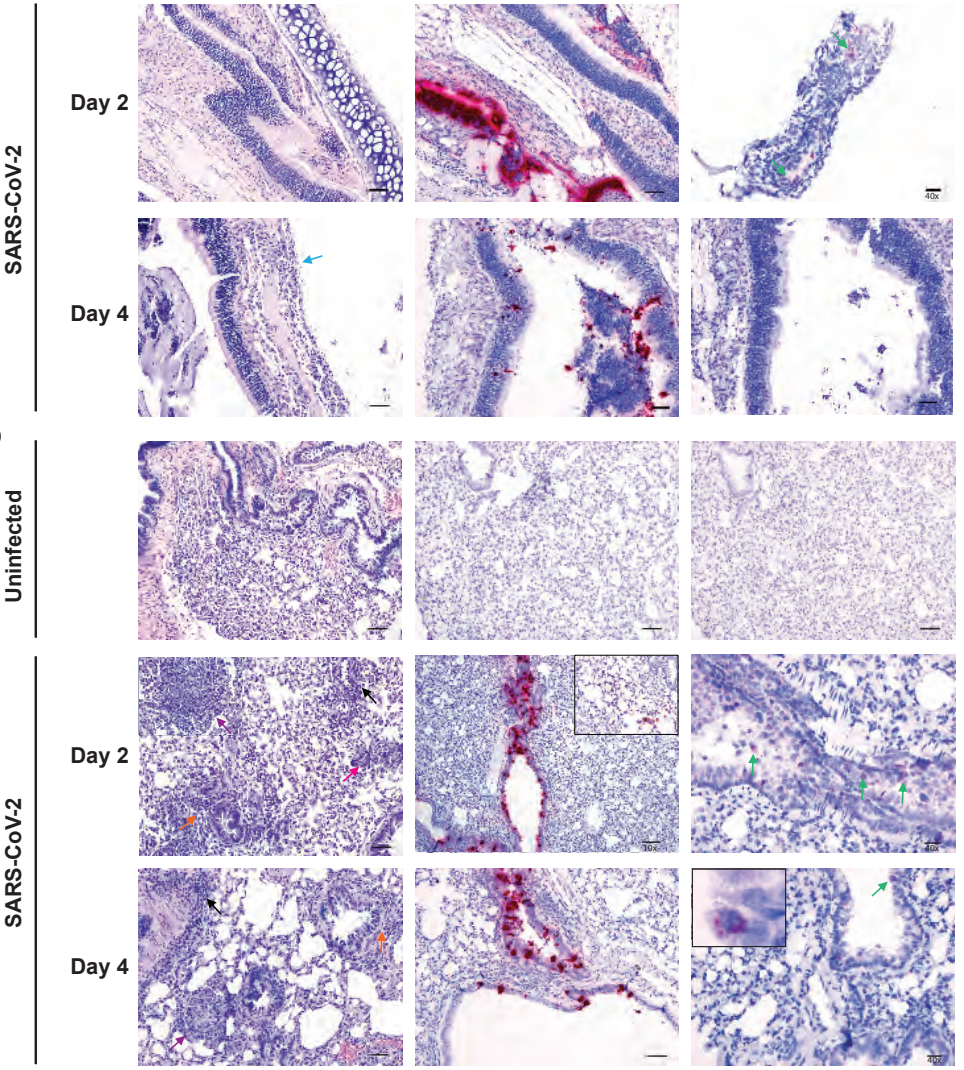

Figure 3: Histopathology and virus distribution. Hematoxylin/eosin (H\&E) staining (left) and in situ hybridization (ISH) using anti-sense probes that detect the SARS-CoV-2 genome/mRNA (middle) and sense probes that detects anti-genomic RNA (right) were carried out on a) nasal turbinates and b) lung tissue of uninfected and SARS-CoV-2-infected deer mice $\left(1 \times 10^{5} \mathrm{TCID}_{50}\right.$ i.n. route) at 2 and $4 \mathrm{dpi}$. Positive detection of viral genomic RNA/mRNA or anti-genomic RNA is indicated by magenta staining (middle/right panels and insets). Arrows indicate perivascular infiltrations of histiocytes and neutrophils (black), peribronchiolar infiltrations of histiocytes and neutrophils (orange), mild neutrophilic infiltration in the submucosa (blue), occasionally observed discrete foci of interstitial pneumonia (purple), occasionally observed multinucleated syncytial cells (magenta), and anti-genomic RNA occasionally in individual scattered bronchiolar epithelial cells (green and right inset). The magnification is 20x for H\&E and 20x for ISH unless otherwise indicated. Scale bars $=100 \mu \mathrm{m}, 50 \mu \mathrm{m}$, and $20 \mu \mathrm{m}$ for $10 \mathrm{x}$, $20 \mathrm{x}$, and $40 \mathrm{x}$, respectively. 
a
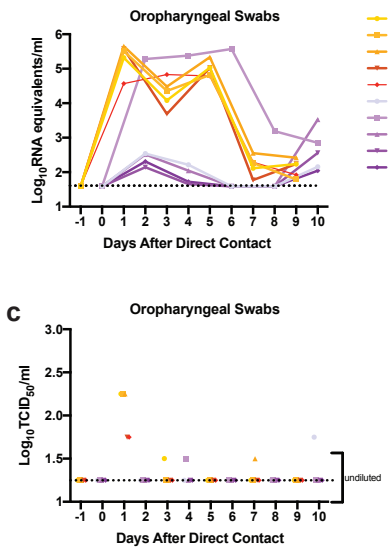

e

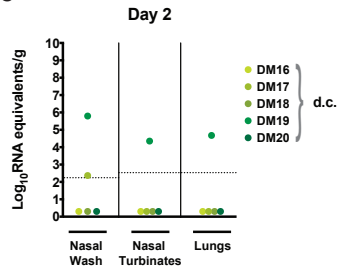

h
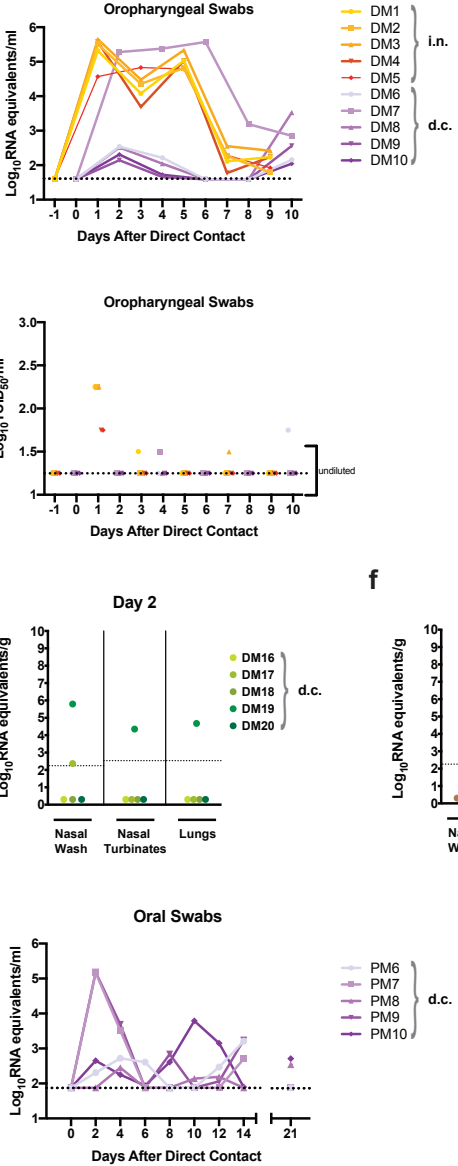

f

b
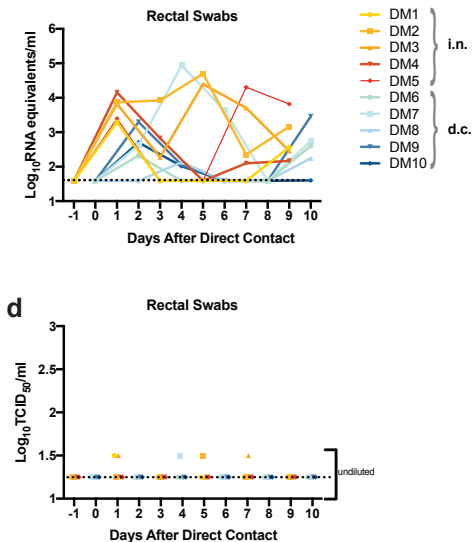

Day 4

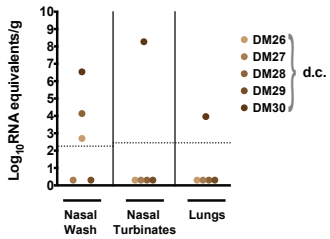

g

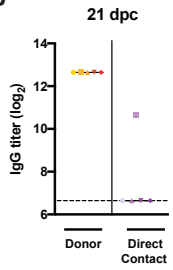

Rectal Swabs

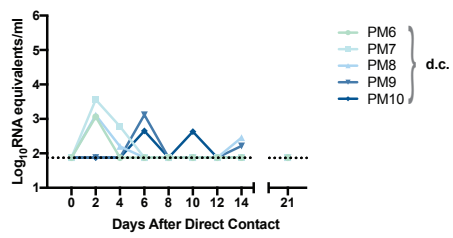

Figure 5: Transmission of SARS-CoV-2 between deer mice through direct contact. a-g, Adult male and female deer mice were exposed to $10^{6}$ TCID $_{50}$ SARS-CoV-2 by an i.n. route of infection. At 1 dpi individual inoculated donor deer mice were transferred to a new cage and co-housed with a single naïve deer mouse (1:1 ratio) to assess SARS-CoV-2 transmission by direct contact. Deer mice were either maintained in direct contact throughout the 10 day study for serial swabbing or humanely euthanized on 2 or 4 days post-contact (dpc) for tissue collection. a-d, Viral RNA levels (a, b) and infectious viral loads (c, d) were measured in oropharyngeal swabs and rectal swab samples every other day from -1 to 10 days after direct contact was initiated. Oropharyngeal or rectal swab samples obtained from the same animal are demarcated with a unique colour, and samples derived from co-housed pairs share the same symbol. e-f, An additional transmission study was carried out in the same manner and nasal wash, nasal turbinates, and lung samples were collected from contact animals at (e) $2 \mathrm{dpc}$ and (f) $4 \mathrm{dpc}$. g, IgG antibody response (reciprocal serum dilution) against SARS-CoV-2 S and $\mathrm{N}$ in donor and direct-contact exposed animals were

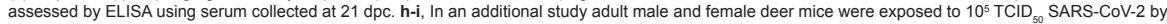
an intranasal route of infection. At 2 dpi individual inoculated donor deer mice were transferred to a new cage and co-housed with a single naïve deer mouse (1:1 ratio) to assess SARS-CoV-2 transmission by direct contact $(n=5)$. Viral RNA levels were measured in (h) oropharyngeal swabs and (i) rectal swab samples every other day from 0 to 14 days after direct contact was initiated, and final swab samples were collected at $21 \mathrm{dpi}$ and assessed. Dotted or dashed lines indicate the limit of detection. Bars indicate means. Data were collected from two independent experiments. 